\title{
Zero Distribution of System with Unknown Random Variables Case Study: Avoiding Collision Path
}

\author{
Setyamartana Parman ${ }^{1}$, Affiani Machmudah ${ }^{1 \mathrm{a}}$, and M. B. Baharom ${ }^{1}$ \\ ${ }^{1}$ Universiti Teknologi PETRONAS, Mechanical Engineering Department, Bandar Seri Iskandar, \\ Tronoh, Perak, Malaysia
}

\begin{abstract}
This paper presents the stochastic analysis of finding the feasible trajectories of robotics arm motion at obstacle surrounding. Unknown variables are coefficients of polynomials joint angle so that the collision-free motion is achieved. $\widetilde{a}_{k}$ is matrix consisting of these unknown feasible polynomial coefficients. The pattern of feasible polynomial in the obstacle environment shows as random. This paper proposes to model the pattern of this randomness values using random polynomial with unknown variables as coefficients. The behavior of the system will be obtained from zero distribution as the characteristic of such random polynomial. Results show that the pattern of random polynomial of avoiding collision can be constructed from zero distribution. Zero distribution is like building block of the system with obstacles as uncertainty factor. By scale factor $k$, which has range, the random coefficient pattern can be predicted.
\end{abstract}

\section{Introduction}

Modeling complex systems as random polynomial presents in many cases mathematics, engineering and physics. Pioneer works in the random polynomial has been conducted by Littlewood et al [1, 2]. Fifty years after the work of M. Kac [4], Sheep et al [5] showed that when the polynomials degree $\mathrm{N}$ gets large, the zeros of random polynomial tend to concentrate near the unit circle in the complex plane. Nowadays, the random polynomial is very fascinating to be investigated since the distribution of zero of polynomials of high degree with random coefficients present in quantum chaotic dynamics [7]. Research of random polynomial application has been introduced into communication engineering by [8]. They discussed the density of the zeros of a random polynomial with nonzero mean correlated Gaussian coefficients.

In this paper, sixth degree polynomial is utilized as the joint angle path of arm robot motion in the obstacle environment. Different with conventional approach of avoiding collision of robotics arm where it needs to find the end-effector trajectories first before converted to joint angle trajectories, the avoiding collision random polynomial modeling will find the joint angle trajectories directly without searching the end-effector trajectories..

The joint angle matrix is the matrix composed from the joint trajectory of each link. This matrix is feasible if and only if the motion caused by it is collision-fee. Due to obstacle position, not all values of the real numbers to compose the joint angle matrix will make the feasible motion. It means the polynomial coefficient matrix is composed from proper values of the real number. Machmudah et al

${ }^{\text {a }}$ Corresponding author : de_affela@yahoo.com

This is an Open Access article distributed under the terms of the Creative Commons Attribution License 2.0, which permits unrestricted use, distribution, and reproduction in any medium, provided the original work is properly cited. 
[9] use Genetic algorithm (GA) and Particle Swarm Optimization (PSO) in finding the feasible coefficient polynomial. Although the GA and PSO can be used to find this feasible coefficient and show good performance in evolving it; however, the pattern of avoiding collision polynomial coefficient is still unknown, except just random. Preliminary investigation of the difficulty to find the pattern of the feasible joint angle matrix has been presented in [10]. This paper will use zero distribution of random unknown variables to model the pattern of collision-free polynomial of arm robot motion.

\section{Polynomial joint angle of arm robot motion in the proximity of obstacles}

Using polynomial as joint angle is very essential in the arm robot motion since the polynomial will give smooth and continuous trajectories. Without obstacles, finding polynomial joint angle is deterministic problem where no randomness involved in the system.

When obstacles are suddenly placed in the environment, finding polynomial joint angle path will not be simple as system without obstacles. There is a requirement, collision-free which should be achieved for all motion. The collision-free polynomial coefficients need to be searched. This paper will use the polynomial degree sixth as avoiding collision joint angle path as used in [9].

Polynomial degree sixth of avoiding collision is defined in the following

$$
\begin{gathered}
\theta_{n k}=a_{6 k} r^{6}+a_{5 k} r^{5}+a_{4 k} r^{4}+a_{3 k} r^{3}+a_{0 k} \\
a_{o k}=\theta_{n k} ; a_{1 k}=a_{2 k}=0 ; a_{5 k}=-3 a_{6 k}-6 \theta_{i k}+6 \theta_{f k} \\
a_{4 k}=0.5\left(-9 a_{6 k}-5 a_{5 k}\right) \quad ; a_{3 k}=\theta_{f k}-\theta_{i k}-a_{6 k}-a_{5 k}-a_{4 k}
\end{gathered}
$$

where $\theta_{i k}, \theta_{f k}, a_{n k}$, and $r$ are the initial joint angle of $k^{\text {th }}$ link, the final joint angle of $k^{\text {th }}$ link, the $n^{\text {th }}$ polynomial coefficient of $k^{\text {th }}$ link, and the linear time scale respectively .

Boundary conditions of this avoiding collision polynomial are known initial joint angle, known final joint angle, zero values of the initial velocity, the final velocity, and the initial and final accelerations. Figure 1 shows the pattern of the joint angle trajectories for different $a_{6 k}$. Due to linear time scale, the value of $r$ used during arm robot motion is limited at $[0,1]$ only.

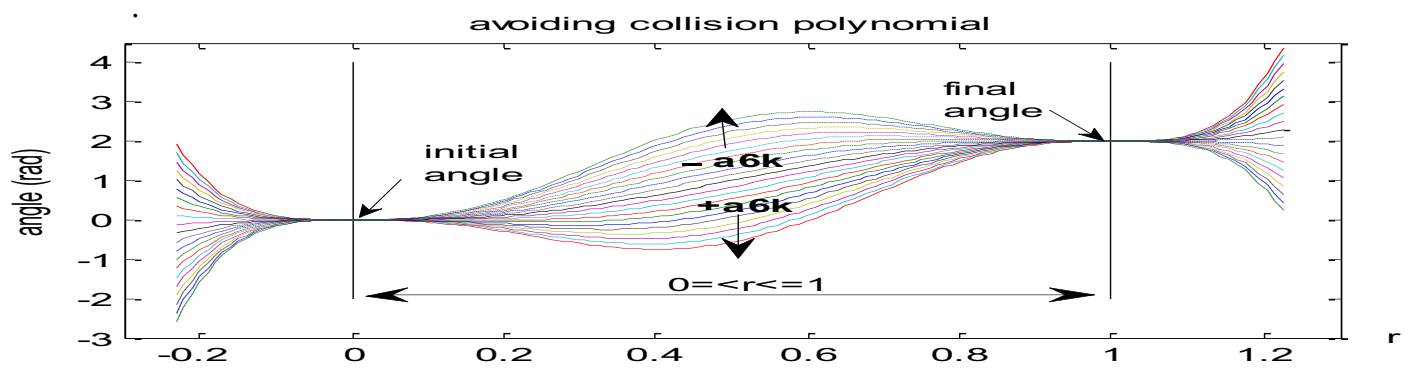

Figure 1. The possible joint angle pattern from all possible real number

\section{Random polynomial coefficients of avoiding collision path}

\section{$3.1 \tilde{a}_{k}$ matrix}

For each link, avoiding collision polynomial has one unknown coefficient, $a_{6 k}$, which is modeled as the random real number. For n-link, there will be $\mathrm{n} a_{6 k}$.

The feasible matrix of unknown polynomial coefficients can be defined as follows

$$
\widetilde{a}_{k}=\left[\begin{array}{llll}
\widetilde{a}_{61} & \widetilde{a}_{62} & \ldots & \widetilde{a}_{6 k}
\end{array}\right]
$$


where $\tilde{a}_{k}$ and $\tilde{a}_{6 k}$ are the feasible matrix of polynomial coefficients and the proper leading coefficients of the avoiding collision polynomial, respectively.

\subsection{Zero Distribution of avoiding collision random polynomial}

Previous researches have been shown that zero of random polynomial can model the chaotic system $[5,7]$. It will be very essential to investigate the zero distribution of the avoiding collision path since the obstacles has transform the deterministic polynomial joint angle path into random polynomial.

It is generally known that zero distribution of large degree of random polynomials in the complex plane tends to concentrate in unitary circle [1-8], as shown in Figure 2a. The avoiding collision random polynomial has $n$ unknown variables which is small degree polynomial. Preliminary investigation of random pattern of avoiding collision problem using polynomial function has been investigated in [10].

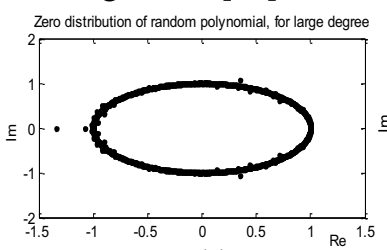

(a)

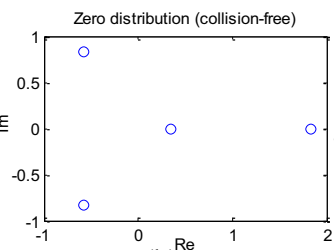

(b)

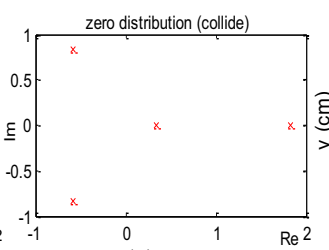

(c)

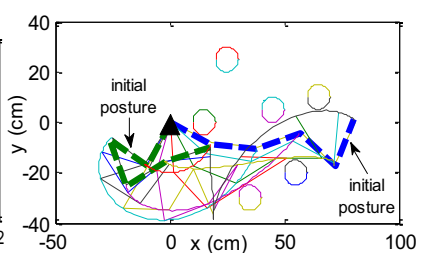

(d)

Figure 2. (a) zero distribution for large degree of random polynomial (b) zero distribution of collision-free polynomial (c) zero distribution for collide random polynomial (d) path and environment

To illustrate the zero distribution of avoiding collision path, 5-DOF planar series robot will be used. Figure $2 \mathrm{~b}$ shows an example of the zero distribution of collision-free polynomial while Figure 2c shows the zero distribution of unfeasible polynomial for five links series robot. The avoiding collision path results and the environment are shown in Figure $2 \mathrm{~d}$. The arm robot has four links so that there are five unknown variables. Thus, the system is equivalent with random polynomial degree four. There will be four possible zeros which can be four real values or two real value and two complex conjugates or four complex conjugates. Figures $2 \mathrm{a}$ and $2 \mathrm{~b}$ show that both of feasible and unfeasible coefficients have similar zero distribution so that it will be difficult to predict the feasible random coefficient from this phenomenon.

\subsection{Proposed method}

Previous section has been shown that there is possibility that the random coefficient of collisionfree and collide joint angles are tend to sit in the similar area. Then, it seems difficult to predict the pattern of avoiding collision polynomial by this zero distribution. The zero distribution shows no difference between unfeasible polynomial and collision-free polynomial.

Instead of analyzing the behavior of random coefficient to get zero distribution, we propose to model zero as basic of building construction of the system.

For $n$ unknown variables, a normalized polynomial degree $n-1$ can be calculated as follows

$$
P_{N}=\left[\left(r-z_{1}\right)\left(r-z_{2}\right) \ldots\left(r-z_{n-1}\right)\right]
$$

where $z_{n}$ are $n^{\text {th }}$ zero of normalized polynomial.

The normalized coefficients are as follows

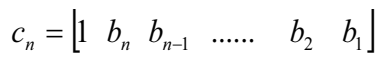

where $c_{n}$ and $b_{n}$ are the normalized coefficient and their values in descending order, respectively

The first column of $c_{n}$ is always 1 . 
This paper proposes to convert the normalized coefficient into the actual coefficient using the following

$$
\widetilde{a}_{k}=\left[c_{n}\right] \widetilde{k}
$$

where $\tilde{a}_{k}$ and $\tilde{k}$ are the feasible unknown random variable and a feasibility factor, respectively.

The collision-free polynomial coefficients will have pattern in the following

$$
\left[\begin{array}{llll}
\widetilde{a}_{61} & \widetilde{a}_{62} & \ldots & \widetilde{a}_{6 k}
\end{array}\right]=\left[c_{n}\right] \widetilde{k}
$$

By this approach, $\tilde{a}_{k}$ is predicted from zero distribution of the normalized coefficient with scale factor $\tilde{k}$. The random coefficient of the system will be developed from zero distribution. It means we will picture the unknown variables as random polynomials. It should be noted that this random polynomial is not random polynomial of joint angle path but it is the random polynomial of the system. Random polynomial characteristic is also present in the Random Matrix Theory (RMT), thus we should investigate whether we can use it in system with n unknown variables. Different with RMT which use polynomial with large degree or $n \rightarrow \infty$, for system with $n$ unknown variables, the zero distribution is $n$ degree only and the zero distribution will not tend to unitary circle since it has few zeros only.

\section{Numerical Experiment}

The proposed method will be applied to predict the pattern of $\widetilde{a}_{k}$ for 3-DOF planar robot in [9] and 5-DOF planar robot in very crowded obstacles.

Table 1. 3-DOF results. Case study: case III from [9]

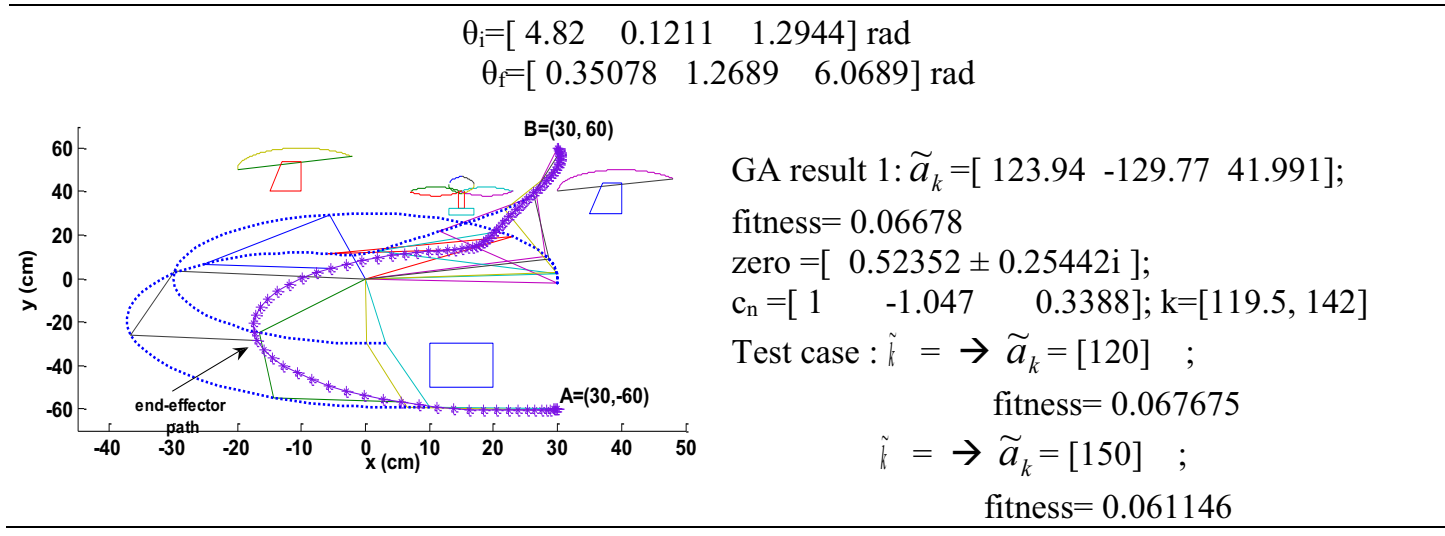

For 3-DOF planar robot, the proposed method has succeeded to predict the collision-free polynomial coefficient. With three unknown variables, there will be two possible zeros which can be either two real values or two complex conjugates. The predicted $a_{k}$ from various zero of random generated variables are shown in Table 1 . The value of $\tilde{k}$ is searched by checking the interval and doing the collision detection at this interval. To avoid the collision with the base, the second and third links should have minimum radius $5 \mathrm{~cm}$ from the base.

Next simulation will use 5-DOF planar robot with very crowded obstacles. The fitness function used is minimum joint angle traveling distance and minimum Cartesian distance with weighting factor 0.5 . Table 2 show the results of pattern predicted from zero distribution. Like 3-DOF planar robot, for 5-DOF planar robot with very crowded obstacles, the zero distribution shows a success to be used as 
basic pattern of collision-free polynomial of the arm robot motion. The system can be model as random polynomial with proper composition of zeros and $\tilde{k}$.

This paper also investigates that if we consider generating polynomials randomly, the value of $\tilde{k}$ is very difficult be found. In this case, the zeros of chosen random polynomial are not proper. In case of collision-free, the pattern of avoiding collision polynomial can be model as proposed approach. This paper uses the computational approach in $[9,10]$, as benchmark case to predict the randomness of avoiding collision polynomial using the proposed method.

Table 2. 5-DOF results

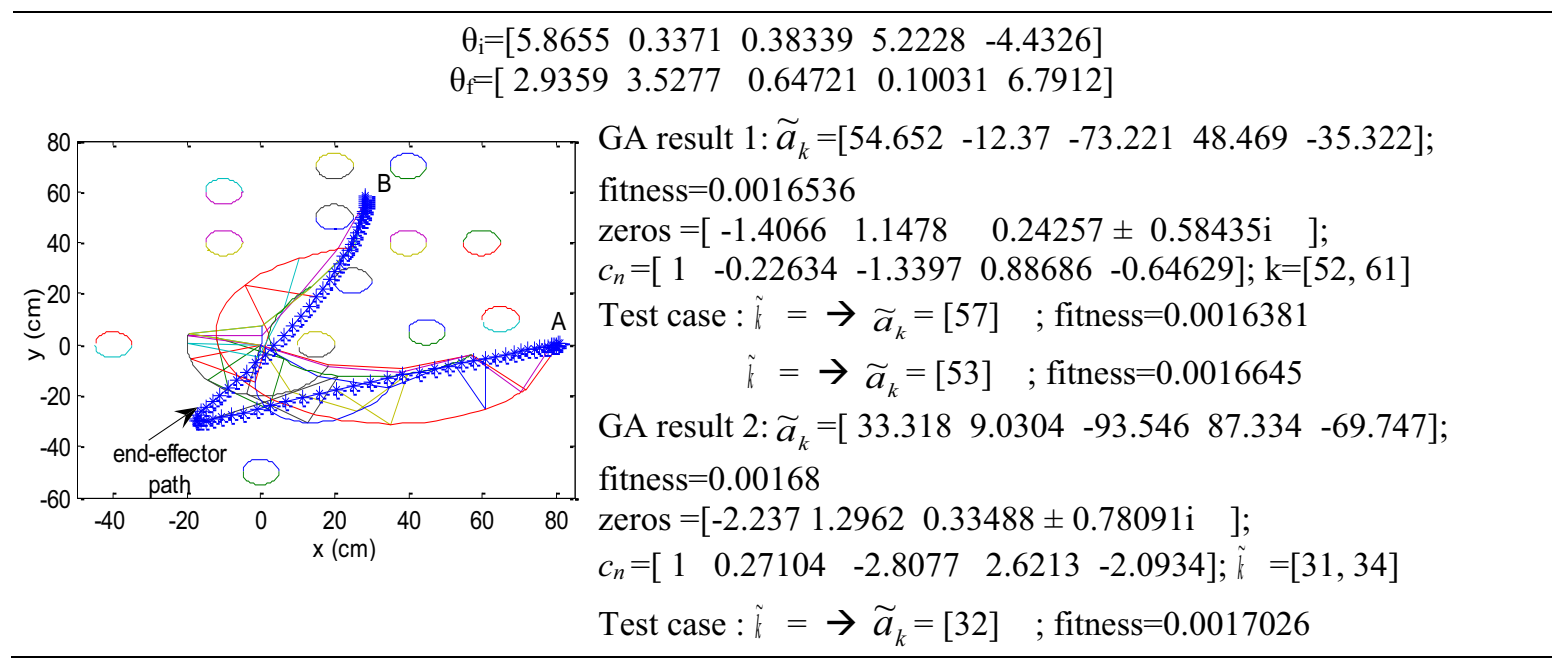

\section{Conclusion}

The proposed method to predict the pattern of collision-free polynomial of arm robot motion has been presented. The random polynomial is constructed from the normalized random coefficient by factor $\tilde{k}$

. Depending on the position of obstacles, the value of $\tilde{k}$ will have specific interval.

\section{References}

1. J. E. Littlewood, A. C. Offord, J. London Math. Soc., 13, 288-295 (1938)

2. J. E. Littlewood, A. C. Offord, Proc. Camb. Phil. Soc., 35, 133-148 (1939

3. J. M. Hammersley, Third Berkley Symp. on Probability and Statistics, pp. 89-111 (1956)

4. M. Kac, Bull. Amer. Math. Soc., 49, 314-320 (1943)

5. L. Shepp, R. J. Vanderbei, Amer. Math. Soc. 347 , 4365-4383 (1995)

6. A. Edelman, E. Kostlan, Bull. Amer. Math. Soc., 32, 1-3 (1995)

7. E. Bogomolny, O. Bohigas, and P. Leboeuf, J. Stat. Phys., 85, 639-679 (1996)

8. R. Schober, , IEEE Trans. Comm., 50, 892-896 (2002)

9. A. Machmudah, S. Parman, A. Zainuddin, S. Chacko, , App. Soft. Comp., 13, 1099-1109 (2013)

10. A. Machmudah, S. Parman, Feasible Joint Angle Continuous Function of Robotics Arm in Obstacles Environment using Particle Swarm Optimization I. Zelinkha (Eds.), Handbook of Optimization From Classical to Modern Approach, Intelligent Systems Reference Library, Springer Verlag, 2013, pp 1047-1071 